\title{
Effect of Different Ovulation Induction Regimens on Pregnancy Outcome of Patients with Endometriosis and Infertility Treated with In vitro Fertilization and Transplantation
}

\author{
YU-HONG XIAO AND YUE-XIN YU* \\ Department of Reproductive Medicine, General Hospital of Northern Theater Command, Liaoning-110016, China
}

\begin{abstract}
Xiao et al.: Buqi Ziyin Combined with Traditional Chinese Medicine Nursing on Thyroid Cancer Patients
To explore the effect of different ovulation induction regimens on pregnancy outcome of patients with endometriosis and infertility treated with in vitro fertilization and transplantation. From December 2016 to December 2019, a total of 324 patients with endometriosis and infertility were selected as subjects. After the same in vitro fertilization treatment, three protocols (super long protocol, modified long protocol and antagonist protocol) were studied retrospectively. Baseline, total Gonadotropin dosage, Gonadotropin days, Luteinizing hormone, progesterone and follicle stimulating hormone levels $(\mathrm{U} / \mathrm{L})$, oocyte retrieval and embryo status, clinical pregnancy rate, live birth rate and abortion rate were compared among the three groups. The total number of days and total amount of Gonadotropin in the super long protocol group were lower than those in the modified long protocol group and antagonist group $(p<0.05)$. The progesterone level before oocyte retrieval was the lowest in the modified long protocol group, followed by the super long protocol group, and the highest in the antagonist group $(\mathbf{p}<0.05)$. The number of retrieved oocytes, mature oocytes and normal cleavage in the modified long protocol group were significantly higher than those in the super long protocol group $(p<0.05)$, but there was no significant difference in the number of excellent embryos $(p>0.05)$. In addition, the number of retrieved ovums, mature ovums, normal cleavage and excellent embryos in the modified long protocol group was the highest $(\mathbf{p}<0.05)$, followed by the super long protocol group $(p<0.05)$. Compared with the other two groups, the pregnancy rate and live birth rate were significantly increased $(p<0.05)$ and the abortion rate was significantly decreased $(p<0.05)$. Finally, the pregnancy rate, live birth rate and abortion rate of the modified long protocol group were higher than those of the antagonist protocol group. The pregnancy rate and live birth rate of super long protocol were higher than those of modified long protocol and antagonist protocol and the abortion rate was lower. The total number of Gonadotropin days and the total amount of Gonadotropin in super long protocol group were lower, which had the advantages of clinical significance. It was a kind of ovulation induction formula worthy of promotion for patients with endometriosis and infertility casen.
\end{abstract}

Key words: Endometriosis, infertility, in vitro fertilization, ovulation induction program

Endometriosis (EMT) is a kind of malignant tumor characteristic of endometrial tissue, which is implanted in the cervical area, and has the characteristics of invasion, distant metastasis and recurrence ${ }^{[1]}$, the incidence rate of women in childbearing age is high, about $10 \% \sim 15 \%{ }^{[2]}$. Endometriosis seriously affects the normal life of patients, the focus of recurrent periodic bleeding, cysts, dysmenorrhea and pelvic pain and other symptoms, and prone to infertility ${ }^{[3]}$. The mechanism of EMT affecting female reproductive function is complex and its mechanism is not clear.
How to improve the pregnancy outcome of EMT patients with infertility has become a worldwide research hotspot. At present, the main methods include drugs, surgery and in vitro fertilization and embryo transfer (IVF-ET). Gonadotropin releasing hormone agonist (GnRHa) is commonly used in clinic. However, GnRHa cannot cure endometriosis, and can cause excessive ovarian suppression. It is still controversial whether GnRHa can improve ovum quality and clinical pregnancy outcome. Laparoscopic surgery has become

*Address for correspondence

E-mail: cheng973862@163.com 
the first-line treatment for endometriosis. It can be used for the diagnosis and staging of endometriosis, the removal of ectopic lesions and the normalization of pelvic structure. However, there is a risk of recurrence of ectopic cysts after surgery ${ }^{[4]}$.

European Society of human reproduction and Embryology guidelines recommend assisted reproductive technology for patients with endometriosis and infertility ${ }^{[5]}$. In vitro fertilization (IVF) can improve the excellent embryo rate and pregnancy outcome of patients with endometriosis ${ }^{[6]}$. Ovulation induction program is one of the decisive steps for IVF success. It is very important to control ovulation induction technology, which is closely related to improving pregnancy outcome. In order to provide basis and reference for clinical research, this paper compared the pregnancy outcomes of IVF treatment in patients with endometriosis and infertility by three ovulation induction regimens: modified long protocol, ultra-long protocol and antagonist protocol.

\section{MATERIALS AND METHODS}

\section{General information:}

From December 2016 to December 2019, 324 patients with endometriosis and infertility were selected as subjects. The age was $(32.46 \pm 3.32) \mathrm{y}$, the duration of infertility was $(4.24 \pm 3.07) \mathrm{y}$ and the body mass index (BMI) was (22.20 \pm 3.35$)$.

Inclusion criteria-In line with the EMT diagnostic criteria of the Chinese Medical Association of Obstetrics and Gynecology; patients and their families are informed and voluntarily sign the research consent; whether the woman's uterus can bear pregnancy; Age 22-45 y; those who have fertility needs and have not been pregnant for at least one year without contraceptive measures.

Exclusion criteria-Either male or female suffered from serious mental illness; either male or female suffered from acute infection of urogenital system and sexually transmitted diseases; poor compliance, unable to receive IVF treatment and timely review; hydrosalpinx with impact on IVF pregnancy outcome, $\geq 4 \mathrm{~cm}$ uterine leiomyoma, severe adenomyosis; spouse with infertility; parallel participants in other clinical studies.

In this study, patients were divided into three groups according to different ovulation induction protocols, including super long protocol group, modified long protocol group and antagonist protocol group. This study met the requirements of medical ethics and was approved by the ethics committee of our hospital.

Drugs: Long acting triptorelin acetate (Bayi, 3.75 mg/tablet, Lizhu Pharmaceutical Group Co., Ltd., Guoyao Zhunzi H20093852). Triptorelin acetate for injection $(3.75 \mathrm{mg} /$ tube, daphrin, French biotechnology company, Guoyao Zhunzi H20030578). Human chorionic gonadotropin (Shanghai first biochemical pharmaceutical Co., Ltd., Guoyao Zhunzi H31020520). Recombinant follicle stimulating hormone (guonafen, 75 u/tube, Merck snow Co., Ltd., Registration No. S20160040). Urinary follicle stimulating hormone (lishenbao, $75 \mathrm{u}$ /piece, Lizhu group, Lizhu pharmaceutical factory, Guoyao Zhunzi H20052130). Urinary gonadotropin (hMG, $75 \mathrm{u} /$ tube, Ma'anshan Fengyuan Pharmaceutical Co., Ltd., Guoyao Zhunzi H20045720).

\section{Ovulation induction program:}

Modified ultra-long ovulation induction protocol: the patient received intramuscular injection of long-acting triptoreline acetate on the third day of menstruation.

Modified long protocol: on the first or second day of menstruation, the patient came to the hospital for injection of $3.75 \mathrm{mg}$ of daphnetin. Antagonist regimen: clomiphene citrate $100 \mathrm{mg} / \mathrm{d}$ was taken orally on the third day of menstruation until the trigger day.

\section{Ovulation induction:}

About $30 \mathrm{~d}$ after injection, B-ultrasound and serum hormone were used to detect the ovarian downregulation. When the diameter of the largest follicle is less than $10 \mathrm{~mm}$ and the thickness of endometrium is less than or equal to $5 \mathrm{~mm}$, we can judge that it reaches the down regulation standard and start promoting ovulation. Gonadotropin is injected intramuscularly for ovulation induction. The drugs for ovulation induction include recombinant follicle stimulating hormone, urinary follicle stimulating hormone and urinary gonadotropin. The attending physician adjusts the type and dosage of drugs according to the hormone level and follicle growth of patients, and decides the starting dose of gonadotropin according to the age and body mass index of patients. When the diameter of dominant follicle reached $14 \mathrm{~mm}, \mathrm{GnRH}$ antagonist was injected daily until the day of trigger.

\section{Oocyte retrieval:}

When there are two or more dominant follicles with a diameter greater than or equal to $18 \mathrm{~mm}, 250 \mu \mathrm{g}$ recombinant human chorionic gonadotropin was maceutical Sciences 
injected intramuscularly that night. After $36-38 \mathrm{~h}$, the oocytes were removed by vaginal ultrasound. According to hormone level, embryo condition, endometrium condition and patient's will, ovum freezing or transplantation is decided. Vitrification was used for freezing. The ovums were treated with cryoprotectant and stored in liquid nitrogen. The ovums waiting for transplantation were placed in a $5 \% \mathrm{CO}_{2}$ incubator at $37^{\circ}$.

\section{IVF:}

IVF was performed according to the technical specification for human assisted reproduction ${ }^{[8]}$. On the day of in vitro fertilization, the semen of the man was fully liquefied, and the semen was treated by density gradient centrifugation. After $5 \mathrm{~h}$ of oocytes retrieval, sperm was added into the micro drop of the fertilization dish, and the final concentration of sperm was 250000 pieces of PR grade sperm/ovums, which were cultured overnight in a $5 \% \mathrm{CO}_{2}$ incubator at $37^{\circ}$.

The fertilization was observed 16-20 $\mathrm{h}$ after insemination, and the embryo quality was scored according to the consensus of Istanbul experts in 2001. The evaluation criteria of high-quality embryos were as follows: normal fertilization on the first day, division into 3-5 cells on the second day, more than 7 embryo cells on the third day, and the fragmentation rate was less than $20 \%$. The blastomeres were homogeneous, without vacuoles and multi-nucleation.

Fresh embryos were taken from all the above programs, and all available embryos were preserved by vitrification. According to the patient's menstrual cycle, the endometrial preparation scheme was selected: the natural cycle was selected for those with regular menstruation and normal ovulation; the hormone replacement cycle was selected for those with irregular menstruation or ovulation disorder. Embryo transfer was performed according to the routine freezing and resuscitation in our center. $14 \mathrm{~d}$ after transplantation, blood samples were taken to check the level of human chorionic gonadotropin (hCG). Ultrasound examination was performed 4-5 $\mathrm{w}$ after transplantation. If the gestational sac and heart tube pulsation were found in the uterine cavity, it was confirmed as clinical pregnancy.

\section{Observation index:}

The general conditions of the three groups were compared: age, infertility years, BMI, and embryo transfer number. Ovulation induction indexes were compared among the three groups: total gonadotropin (Gn) dosage, Gn days, luteinizing hormone (LH) level (U/L), progesterone (P) level (mg/L) and follicle stimulating hormone (FSH) level (U/L). The number of retrieved oocytes, mature oocytes, normal cleavage and high-quality embryos were compared among the three groups. Pregnancy diagnosis-blood was drawn $14 \mathrm{~d}$ after transplantation, and $30 \mathrm{~d}$ after transplantation, B-ultrasound was used to determine whether clinical pregnancy, intrauterine and extrauterine conditions, germ and heart tube pulsation were detected, and clinical pregnancy rate, live birth rate and abortion rate were calculated.

\section{Statistical analysis:}

All data were analyzed by SPSS 22.0 software. Continuous variables pass the normality test to express. The count variables were expressed by the number of cases and percentage, and the comparison between groups was performed by chi square test; the test level was bilateral $\alpha=0.05$. $\mathrm{p}<0.05$ was considered statistically significant.

\section{RESULTS AND DISCUSSION}

The average age, infertility years, BMI and embryo transfer number of the three groups were not statistically significant $(\mathrm{p}>0.05)$, indicating that the three groups were comparable, as shown in Table 1.

According to the statistics of IVF of three groups, we found that the total number of GN days and the total number of GN in the super long protocol group were lower than those in the modified long protocol group and the antagonist group $(\mathrm{p}<0.05)$, and there was no significant difference in the total number of GN days and the total number of GN between the modified long protocol group and the antagonist protocol group $(p>0.05)$. For $p$ level before oocyte retrieval, the modified long protocol group was the lowest, followed by the super long protocol group, and the antagonist group was the highest $(\mathrm{p}<0.05)$, as shown in Table 2 .

The number of retrieved oocytes, mature oocytes and normal cleavage in the modified long protocol group were significantly higher than those in the super long protocol group $(p<0.05)$. The number of retrieved oocytes, mature oocytes, normal cleavage and excellent embryos in the modified long protocol group were higher than those in the antagonist protocol group $(p<0.05)$; the number of retrieved oocytes, mature oocytes and normal cleavage in the super long protocol 
group were higher than those in the antagonist protocol group ( $\mathrm{p}<0.05$ ), as shown in Table 3 .

Statistics of pregnancy outcomes of the three groups showed that the pregnancy rate and live birth rate of the super long protocol group were significantly higher than those of the modified long protocol group and the antagonist protocol group $(\mathrm{p}<0.05)$, and the abortion rate was significantly lower than that of the other two groups $(\mathrm{p}<0.05)$. The pregnancy rate and live birth rate of the modified long protocol group were higher than those of the antagonist protocol group, and the abortion rate was lower $(\mathrm{p}<0.05)$, as shown in Table 4 .

Endometriosis is a common clinical gynecological disease in young and middle-aged women. It means that the active endometrial tissue (glands and stroma) is implanted outside the uterus and invades other normal tissues. Its main symptoms and consequences are abdominal pain and infertility. EMT combined with infertility has gradually become an important cause of infertility in women of childbearing age. This disease will not only bring physical and mental damage to women, affect family harmony and happiness, but also increase the economic burden of patients. The pathological and physiological process of endometriosis is complex and changeable, which may be the result of the synergistic effect of pelvic microenvironment and anatomical structure changes, oxidative stress injury, immune inflammatory response, genetic factors and endocrine dysfunction. The method to improve the pregnancy outcome of EMT with infertility has been a hot and difficult point in clinical research. IVF has become an important treatment method to improve the pregnancy outcome of EMT patients. One of the key factors for successful pregnancy is the quality of oocytes obtained by ovulation induction, in which pituitary down regulation is the key process to ensure the quality of oocytes ${ }^{[11]}$.

In this study, a retrospective analysis was used to compare the embryo and pregnancy status of 324 patients with endometriosis and infertility. Our

TABLE 1: COMPARISON OF BASIC DATA OF THREE GROUPS OF PATIENTS ( $\overline{\mathbf{x}} \pm \mathbf{S})$

\begin{tabular}{lcccc}
\hline Groups & Average age & Years of infertility & BMI & Number of embryo transfer \\
\hline Modified long protocol & $34.63 \pm 2.86$ & $4.24 \pm 3.07$ & $22.43 \pm 3.12$ & $1.84 \pm 0.52$ \\
Ultra-long protocol & $33.41 \pm 3.09$ & $4.17 \pm 3.14$ & $22.06 \pm 3.32$ & $1.79 \pm 0.43$ \\
Antagonist regimen & $32.48 \pm 3.30$ & $4.25 \pm 3.17$ & $22.45 \pm 3.52$ & $1.86 \pm 0.58$
\end{tabular}

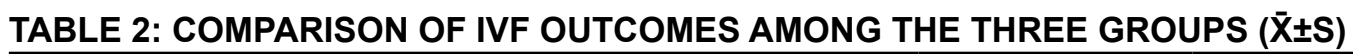

\begin{tabular}{lccccc}
\hline Groups & Total Gn days & Total Gn & $\begin{array}{c}\text { LH level before } \\
\text { oocyte retrieval } \\
(\mathrm{U} / \mathrm{L})\end{array}$ & $\begin{array}{c}\text { P level before } \\
\text { oocyte retrieval } \\
(\mathrm{mg} / \mathrm{L})\end{array}$ & $\begin{array}{c}\text { FSH level before } \\
\text { oocyte retrieval } \\
(\mathrm{U} / \mathrm{L})\end{array}$ \\
\hline Modified long protocol & $9.98 \pm 3.12^{\$}$ & $2384.40 \pm 961.33$ & $4.52 \pm 1.32$ & $0.52 \pm 0.15^{\# \$}$ & $9.83 \pm 4.40$ \\
Ultra-long protocol & $7.13 \pm 2.08^{* \#}$ & $2060.83 \pm 733.89$ & $4.31 \pm 2.67$ & $0.73 \pm 0.18^{* \#}$ & $10.24 \pm 4.12$ \\
Antagonist regimen & $9.55 \pm 2.22^{\$}$ & $2349.40 \pm 949.26$ & $4.09 \pm 2.11$ & $0.84 \pm 0.27^{\$ *}$ & $9.31 \pm 3.45$ \\
\hline
\end{tabular}

Note: "means $p<0.05$ vs. Modified long protocol; "means $p<0.05$ vs. Antagonist regimem; ${ }^{\text {m } m e a n s ~} p<0.05$ vs. Ultra long protocol

TABLE 3: COMPARISON OF OOCYTE RETRIEVAL AND EMBRYO RETRIEVAL AMONG THREE GROUPS $(\overline{\mathrm{X}} \pm \mathrm{S})$

\begin{tabular}{lccccc}
\hline Groups & $\mathrm{N}$ & $\begin{array}{c}\text { Number of } \\
\text { oocytes }\end{array}$ & $\begin{array}{c}\text { Number of mature } \\
\text { oocytes }\end{array}$ & $\begin{array}{c}\text { Normal oocytes } \\
\text { division number }\end{array}$ & $\begin{array}{c}\text { Number of excellent } \\
\text { embryos }\end{array}$ \\
\hline Modified long protocol & 105 & $11.55 \pm 8.25^{\# \$}$ & $10.26 \pm 7.29^{\# \$}$ & $6.42 \pm 5.43^{\# \$}$ & $3.23 \pm 3.19^{\#}$ \\
Antagonist regimen & 62 & $7.21 \pm 5.35 \$^{*}$ & $6.07 \pm 4.83 \$^{*}$ & $3.92 \pm 3.01 \$^{*}$ & $2.19 \pm 2.09^{*}$ \\
Ultra-long protocol & 157 & $9.04 \pm 5.80^{* \#}$ & $8.31 \pm 5.67^{* \#}$ & $5.11 \pm 4.08^{* \#}$ & $2.62 \pm 2.63$ \\
\hline
\end{tabular}

Note: *means $p<0.05$ vs. Modified long protocol; \#means $p<0.05$ vs. Antagonist regimem; \$means $p<0.05$ vs. Ultra long protocol

TABLE 4: COMPARISON OF PREGNANCY OUTCOMES AMONG THE THREE GROUPS [N (\%)]

\begin{tabular}{lcccc}
\hline Groups & N & Gestation & Live birth & Abortion \\
\hline Modified long protocol & 105 & $76(72.4 \%)^{\# \$}$ & $56(53.3 \%)^{\# \$}$ & $20(19.0 \%)^{\# \$}$ \\
Antagonist regimen & 62 & $42(67.7 \%)^{* \$}$ & $25(40.3 \%)^{* \$}$ & $17(27.4 \%)^{* \$}$ \\
Ultra long protocol & 157 & $116(73.9 \%)^{\# *}$ & $103(65.6 \%)^{\# *}$ & $13(8.3 \%)^{\# *}$ \\
\hline
\end{tabular}

Note: *means $\mathrm{p}<0.05 \mathrm{vs}$. Modified long protocol; "means $\mathrm{p}<0.05 \mathrm{vs}$. Antagonist regimem; $\$$ means $\mathrm{p}<0.05 \mathrm{vs}$. Ultra long protocol 
survey results showed that the pregnancy rate and live birth rate of patients treated with ultra-long protocol were higher than those treated with modified long protocol and antagonist protocol, and the abortion rate was significantly lower than those treated with modified long protocol and antagonist protocol, indicating that the ultra-long protocol can significantly improve the pregnancy outcome of patients with endometriosis complicated with infertility The transplantation rate and clinical pregnancy rate of fresh cycle were analyzed. The results of this study also showed that the total number of days of GN and the total amount of GN in the super long protocol group were lower than those in the modified long protocol group and the antagonist group, indicating that the super long protocol was convenient for medication and less injection times, which directly affected the clinical compliance and patient acceptance. The reason may be the decrease of estrogen level in the process of promoting ovulation, which improves endometrial receptivity and avoids ovarian hyper stimulation $^{[12]}$.

The number of retrieved oocytes, mature oocytes and normal cleavage in the modified long protocol group was significantly higher than that in the super long protocol group and the antagonist protocol group. The number of retrieved oocytes, mature oocytes and normal cleavage in the super long protocol group was higher than that in the antagonist protocol group. This study found that the pregnancy rate and live birth rate of modified long protocol were high, and the abortion rate was low ${ }^{[13]}$. GnRH-a is a drug that can compete with endogenous GnRH to act on the pituitary gland, which can make the pituitary GnRH-a receptor in a desensitized state, thus inhibiting the ovarian secretion of related hormones ${ }^{[14]}$. In this study, GnRH-a was used in both modified long protocol and super long protocol to achieve the effect of pituitary down regulation. However, the antagonist scheme also has its clinical advantages. The GN releasing hormone used in this scheme has a wide range of application, avoids the transient increase effect of GnRH-a, does not need the down-regulation process, has short treatment cycle, and is flexible and convenient to use ${ }^{[15]}$.

In conclusion, modified long protocol has clinical advantages in improving the number of IVF oocytes and laboratory embryos, but the pregnancy rate and live birth rate of super long protocol are higher than those of modified long protocol and antagonist protocol, and the abortion rate is significantly lower than that of modified long protocol and antagonist protocol. In addition, the total number of GN days and the total amount of GN in the super long protocol group were lower than those in the modified long protocol group and the antagonist group, which had the advantage of clinical significance. It was a worthy ovulation induction program for patients with endometriosis and infertility.

\section{Acknowledgements:}

This work was supported by the General Hospital of NorthernTheater Command.

\section{Conflicts of interest:}

The authors report no conflicts of interest.

\section{REFERENCES}

1. Zuberi NF, Rehman R. Endometriosis and subfertility. Subfertility 2021;135-46.

2. Tan J, Cerrillo M, Cruz M, Cecchino GN, Garcia-Velasco JA. Early Pregnancy Outcomes in Fresh Versus Deferred Embryo Transfer Cycles for Endometriosis-Associated Infertility: A Retrospective Cohort Study. J Clin Med 2021;10(2):344.

3. Shebl O, Sifferlinger I, Habelsberger A, Oppelt P, Mayer RB, Petek E, et al. Oocyte competence in in vitro fertilization and intracytoplasmic sperm injection patients suffering from endometriosis and its possible association with subsequent treatment outcome: a matched case-control study. Acta Obstet Gynecol Scand 2017;96(6):736-44.

4. Mosbrucker C, Somani A, Dulemba J. Visualization of endometriosis: comparative study of 3-dimensional robotic and 2-dimensional laparoscopic endoscopes. J Robot Surg 2018;12(1):59-66.

5. Haiyu T, Tianmin G. Diagnosis and treatment of endometriosis: Interpretation of the new guidelines of the European Society of human reproduction and Embryology (ESHRE). Chin J Reproduct Health 2015;26(2):176-80.

6. Ylmaz N, Ceran MU, Ugurlu E N. Impact of endometrioma and bilaterality on IVF / ICSI cycles in patients with endometriosis. J Gynecol Obstetrics Human Reproduction 2020:101839.

7. Endometriosis cooperative group, Chinese society of Obstetrics and gynecology. Guidelines for diagnosis and treatment of endometriosis. Chin J Obstetrics Gynecol 2015;10(3):161-9.

8. Ministry of health. Notice of the Ministry of health on revising relevant technical specifications, basic standards and ethical principles of human assisted reproductive technology and human sperm bank. Bulletin of the Ministry of health of the people's Republic of China, 2003;(3):1-10.

9. Evans MB, Decherney AH. Fertility and endometriosis. Clin Obstetrics Gynecol 2017;60(3):497-502.

10. Miller JE, Ahn SH, Monsanto SP, Khalaj K, Koti M, Tayade C. Implications of immune dysfunction on endometriosis associated infertility. Oncotarget 2017;8(4):7138-47.

11. Yin Y, Mao Y, Liu A, Shu L, Yuan C, Cui Y, et al. Insufficient Cumulus Expansion and Poor Oocyte Retrieval in Endometriosis-Related Infertile Women. Reproductive Sci 2021:1-9.

12. Khan Z. Fertility-related considerations in endometriosis. Abdominal Radiol 2020;45:1754-61.

13. Danhof NA, Van Wely M, Koks CA, Gianotten J, De Bruin JP, 
Cohlen BJ, et al. The SUPER study: protocol for a randomised controlled trial comparing follicle-stimulating hormone and clomiphene citrate for ovarian stimulation in intrauterine insemination. BMJ open 2017;7(5):e015680.

14. Sallam HN, Garcia-Velasco JA, Dias S, Arici A, Abou-Setta AM. Long-term pituitary down-regulation before in vitro fertilization (IVF) for women with endometriosis. Cochrane Database of Sys Rev 2006;1(1):CD004635.

15. Chankitisakul V, Pitchayapipatkul J, Chuawongboon P, Rakwongrit D, Sakhong D, Boonkum W, et al. Comparison of three superovulation protocols with or without GnRH treatment at the time of artificial insemination on ovarian response and embryo quality in Thai native heifers. Tropical Animal Health and Production 2017;49(3):633-9.

This is an open access article distributed under the terms of the Creative Commons Attribution-NonCommercial-ShareAlike 3.0 License, which allows others to remix, tweak, and build upon the work non-commercially, as long as the author is credited and the new creations are licensed under the identical terms

This article was originally published in a special issue,

"Evolutionary Strategies in Biomedical Research and

Pharmaceutical Sciences" Indian J Pharm Sci 2021:83(3)

Spl issue;139-144 